\title{
Temporal regulation of lin-14 by the antagonistic action of two other heterochronic genes, lin-4 and lin-28
}

\author{
Prema Arasu, Bruce Wightman, and Gary Ruvkun \\ Department of Molecular Biology, Massachusetts General Hospital; Department of Genetics, Harvard Medical School, \\ Boston, Massachusetts 02114 USA
}

\begin{abstract}
Heterochronic genes form a regulatory pathway that controls the temporal sequence of the Caenorhabditis elegans postembryonic cell lineage. One of these genes, lin-14, encodes a nuclear protein that constitutes a temporal developmental switch. During wild-type development, lin-14 protein is abundant during early larval stage 1 (L1) to specify L1-specific cell lineages but is nearly undetectable at L2 and later stages to specify L2-specific and later cell lineages. To determine the roles played by other genes in executing this temporal switch, we have analyzed how lin-14 expression is regulated by other heterochronic genes. lin-4 is required to down-regulate lin-14 protein levels during the L1 stage, whereas lin-28 positively regulates lin-14 protein levels. The lin-4 gene product is a candidate for interacting with the negative regulatory element in the $3^{\prime}$-untranslated region of lin-14. lin-29 mutations do not affect lin-14 protein levels, consistent with lin-29 acting downstream of lin-14. Switching off lin-14 expression during the L1 stage is not triggered by the passage of time per se but, rather, is normally dependent on feeding or the feeding-dependent initiation of postembryonic cell division.
\end{abstract}

[Key Words: lin-14; lin-4; lin-28; lin-29; heterochronic genes; C. elegans; developmental switch]

Received June 3, 1991; revised version accepted July 22, 1991.

Development in multicellular organisms is controlled by a network of genes that specify the temporal and spatial pattern of cell division and differentiation (Horvitz 1988; Ingham 1988). In Caenorhabditis elegans, heterochronic genes control the temporal identities of specific postembryonic cells: Mutations in these genes cause particular cells to adopt identities normally associated with cells at earlier or later stages of development (Chalfie et al. 1981; Ambros and Horvitz 1984). The heterochronic gene lin14 plays a major role in this control of temporal development: Gain-of-function (gf) mutations that inappropriately increase 1 in-14 activity at late stages impose reiterated larval stage 1 (L1)-specific cell lineages at post-Ll stages, and mutations that decrease lin-14 activity cause blast cells during the Ll stage to express precociously L2-specific cell lineages (Ambros and Horvitz 1984).

Genetic and molecular studies have shown that lin-14 specifies cell identities through a temporal gradient of the lin-14 nuclear protein (Ambros and Horvitz 1987; Ruvkun and Giusto 1989|. lin-14 gene activity and the level of lin-14 protein is high in late embryos and L1 stage animals and decreases rapidly during mid-L1 stage to specify the switch from early to late identities of blast cells. Gain-of-function lin-14 mutations cause inappropriately high levels of 7 in-14 protein at all developmental stages (Ruvkun and Giusto 1989) which, in turn, cause reiterated execution of early cell lineages at late devel- opmental stages. These mutations delete or rearrange negative regulatory sequences in the $3^{\prime}$-untranslated region ( 3 ' UTR) of the lin-14 gene, resulting in the abnormal accumulation of 1 in -14 protein late in development (Wightman et al., this issue). Loss-of-function (lf) lin-14 mutations that produce no lin-14 protein affect the same cell lineages but cause the opposite transformations in cell fate-late cell lineages are precociously executed at early developmental stages.

From genetic analyses several other heterochronic genes have been found to affect the temporal pattern of C. elegans development. The lin-4(e912) mutation, as lin-14(gf) mutations, causes reiteration of Ll-specific lineages in many postembryonic cell lineages. lin-28 mutations affect L2 and later lineages (Chalfie et al. 1981; Ambros and Horvitz 1984). lin-29 controls the switch from L4 to adult stage hypodermal cell identities (Ambros 1989). lin-4 and 1 in-14 also regulate the stage at which the initiation of dauer larvae occurs, whereas lin28 and lin-29 do not (Liu and Ambros 1989). This observation suggests that 1 in -4 and 1 in -14 could regulate more cell lineages or cell types than lin-28 and lin-29. Genetic epistasis studies have ordered these genes into a pathway: lin-4 negatively regulates 1 in -14 and perhaps lin28 , either or both of which, in turn, negatively regulate lin-29.

On the basis of genetic analysis, lin-4, lin-28, and lin- 
29 could participate either in the generation or the interpretation of the lin-14 temporal switch. Here, we explore how the lin-14 temporal switch is regulated by these other heterochronic genes. On the basis of comparison of the cellular and temporal expression pattern of lin-14 in mutants and wild type, we find that lin-28 and lin-4 act antagonistically to regulate lin-14. Conversely, lin-29 mutations do not affect lin-14 levels; thus, lin-29 is a candidate downstream gene that may be regulated by lin-14. We also explored how the down-regulation of lin14 protein is normally triggered. We find that the temporal down-regulation of lin-14 protein levels is not initiated by the passage of time per se but, instead, depends on feeding or the initiation of the postembryonic lineage during the $\mathrm{Ll}$ stage.

\section{Results}

In wild-type animals, the lin-14 protein is expressed at a high level in the nuclei of specific somatic cells of late stage embryos and early L1 larvae, but not at subsequent developmental stages of wild-type animals (Fig. 1a; Ruvkun and Giusto 1989). All late embryos and early- to mid-Ll stage animals show intense lin-14 protein staining in hypodermal, intestinal, body wall muscle, nerve ring, and ventral nerve cord nuclei (Fig. la). During the late Ll stage, hypodermal and intestinal nuclei show little or no immunofluorescence, whereas neuronal and muscle cells show faint lin-14 immunofluorescence (Ruvkun and Giusto 1989). By the L2 stage, lin-14 protein is undetectable in most cell types and only weakly detectable in some neurons (Fig. 1a). Quantitation of immunoblots shows that the level of the lin-14 protein relative to a pharyngeal myosin control decreases $\geqslant 25$-fold from $\mathrm{L} 1$ to L2 (B. Wightman and G. Ruvkun, in prep.).
The heterochronic genes lin- 4 and lin-28

affect the temporal regulation of lin-14

To study the regulatory effects of the genetically interacting genes lin-4, lin-28, and lin-29 on lin-14, we analyzed the temporal and cellular expression patterns of lin-14 protein in these heterochronic mutants.

The lin-4(e912) mutant, as with lin-14(gf) mutants, displays a retarded phenotype with reiteration of $L 1$-specific lineages at L2 and later stages (Chalfie et al. 1981). The Lin-4 retarded phenotype is dependent on lin-14 gene activity (Ambros 1989). These genetic results suggest that lin-4 negatively regulates lin-14, although not necessarily at the level of gene expression. We examined the lin-14 immunostaining pattern in lin-4(e912) to ascertain how lin-4 regulates lin-14. The lin-4(e912) allele appears to be a null mutant based on genetic criteria ( $R$. Feinbaum and V. Ambros, pers. comm.). Anti-lin-14 antibodies detect lin-14 protein in lin-4 Ll animals in all nuclei that are detected in wild type. As judged by immunofluorescence intensity, the level of lin-14 protein was similar to wild type at this stage. Unlike wild type, however, lin-14 protein is detected in nuclei at L2, L3, L4, and adult stages (Fig. 1c). This expression of high lin-14 protein levels after the L1 stage was very similar to that observed in the lin-14(gf) mutants, consistent with the similar lineage defects observed (Ambros and Horvitz 1984). As judged by immunofluorescence intensity, the level of lin-14 protein at post-Ll stages in lin4(e912) animals is about half that in the lin-14(n355gf) mutants (Fig. 1b) and similar to less severe lin-14(n536gf) mutants. As with the lin-14(n536n540) null mutant, no lin-14 protein staining at any stage was observed in a lin-4(e912); lin-14(n536n540) mutant, showing that the late stage staining in a lin-4 mutant is caused by misexpression of the lin-14 gene and not expression of an im-

Figure 1. In situ immunofluorescent detection of lin-14 nuclear protein in wild-type and heterochronic mutants. Specimens were fixed and incubated with affinity-purified anti-lin-14 antibodies, followed by a secondary fluorescein isothiocyanate (FITC)-labeled goat anti-rabbit antibody and 4,6-diamido-2-phenylindole (DAPI) stained to visualize all nuclei. Immunofluorescent photographs were taken with FITC (F) filters to show lin-14 protein staining or DAPI (D) filters to show all nuclei. Representative nuclei of a cell type are identified by arrows; (G) gonad; $(H)$ hypoderm; $(I)$ intestine; $(M)$ body wall muscle; $(N)$ nerve ring; $(V)$ ventral cord neurons. Photomicrographs are generally lateral views, in one of two planes. If a hypodermal cell is pointed out, the plane of focus is lateral and large nuclei are all hypodermal $(\mathrm{H})$ and small nuclei are neuronal $(\mathrm{N})$. If intestinal $(\mathrm{I})$ and ventral cord neurons $(\mathrm{V})$ are labeled, the plane of focus is medial and all large nuclei are intestinal and the line of small nuclei ventrally are the ventral nerve cord and small dorsal nuclei are muscle. The head of the animal is identified in most photomicrographs by the intensely staining nerve ring nuclei $(\mathrm{N})$. (a) (Top) FITC; wild-type early L1 stage (Ll), late L1 stage (unlabeled), and L3 stage (L3) animals. Lateral plane showing bright lin-14 protein staining in most nuclei at early L1, somewhat dimmer staining at late L1, and absence of staining at L3. (Bottom) DAPI; same animals with same plane of focus. We have observed very dim lin-14 protein staining in some neuronal nuclei of L2 and later animals in some preparations (M. Finney, unpubl.). (b) (Top left) FITC; lateral view of lin-14(n355gf) mutant early Ll stage, showing intense staining of hypodermal, ventral nerve cord, and nerve ring nuclei. (Top right) FITC; ventral view of lin-14(n355gf) L2 stage, showing lin-14 protein in the ventral cord neurons and in muscle nuclei. Both gain-of-function mutants show inappropriate accumulation of lin-14 protein in L2 and later stages, although the $n 536$ allele is weaker than the $n 355$ allele and lin-14 protein staining is consistently less intense in lin-14(n536) mutant animals. (c) (Left) FITC; lin-4(e912) Ll stage focused at the midline, showing lin-14 protein staining in the nuclei of ventral cord neurons, intestine, hypoderm, and tail ganglion neurons. (Right) FITC; lateral view of lin-4(e912) L2 stage, showing lin-14 protein in hypodermal and postdeirid neuronal nuclei. (d) (Top left) FITC; lin-28(n719) with three animals, two late stage embryos, and an early Ll stage. All animals show accumulation of lin-14 protein in nerve ring, ventral cord, hypoderm, intestine, and muscle nuclei, like wild type at these early developmental stages. (Top middle) FITC; lin-28(n719) early Ll stage, showing a decrease in lin-14 protein staining in the hypodermal and intestinal nuclei compared with neuronal and body wall muscle nuclei. (Top right) FITC; lin-28(n719) L2 stage, showing no lin-14 protein staining. (e) (Top left) FITC; lin-29(n333) L1 stage that displays lin-14 protein in the same nuclei as wild type. (Top right) FITC; lin-29(n333) L2 stage, showing no lin-14 staining. 


\section{(a) WT}

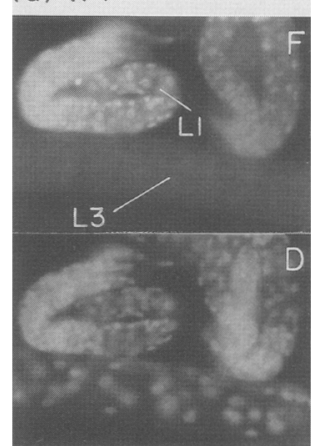

(b) $\operatorname{lin}-14(n 355)$

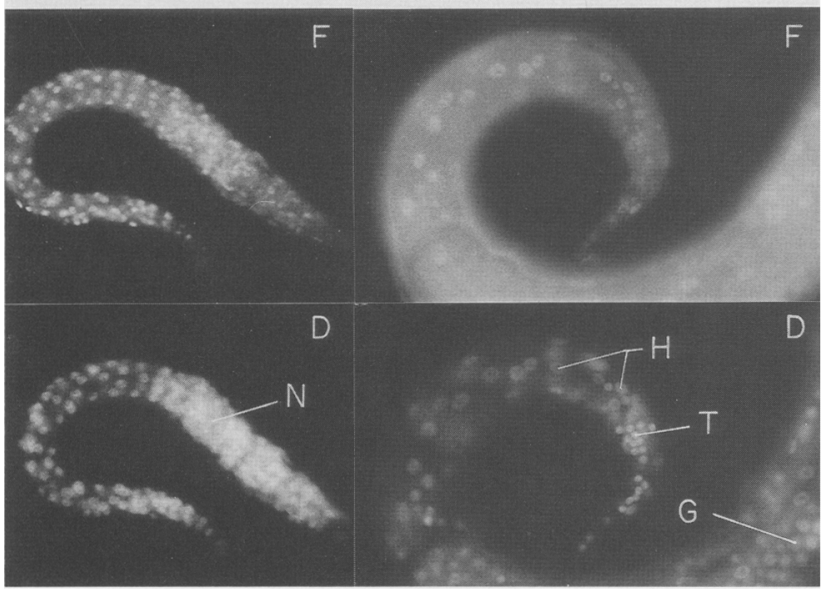

(c) $\operatorname{lin}-4$

(d) $\operatorname{lin}-28$

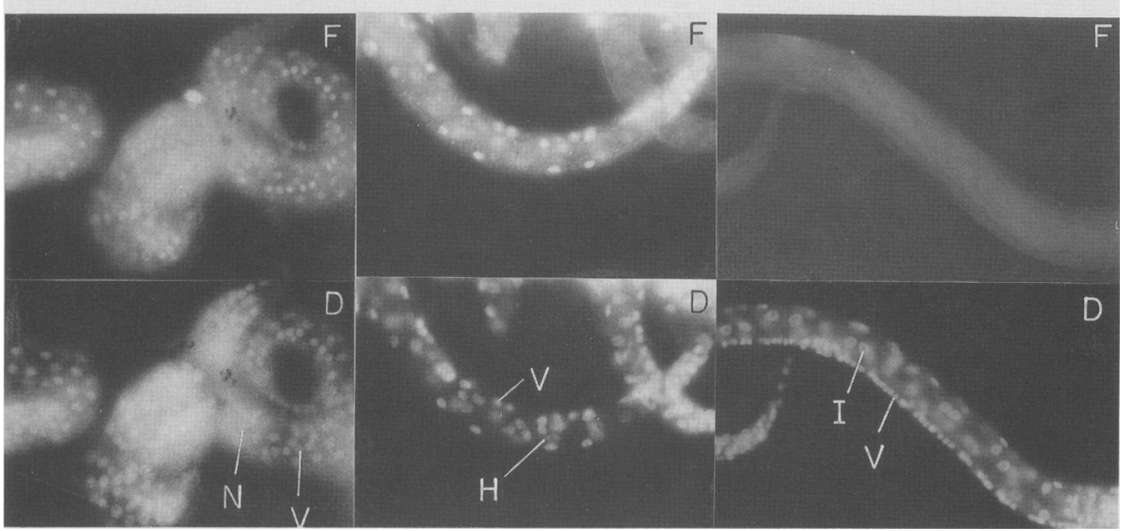

(e) $\operatorname{lin}-29$

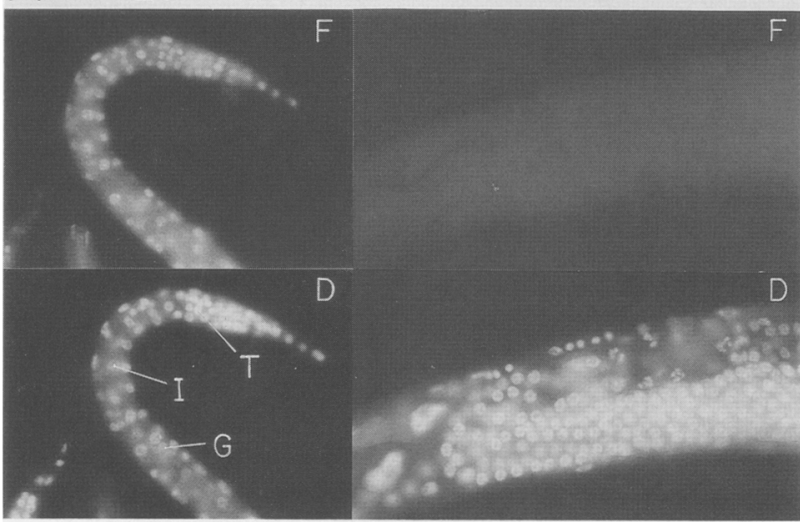

Figure 1. (See facing page for legend.) 
munologically related protein (data not shown). These data show that lin- 4 regulates lin-14 gene activity by controlling the level of the lin-14 protein.

In contrast to lin-4, lin-28 null mutations result in precocious expression of L3 or L4 stage-specific cell lineages in hypodermal blast cells during the L2 stage after a normal L1 stage-specific cell lineage (Ambros and Horvitz 1984). Genetic epistasis analysis showed that lin-28 acts downstream of lin-4 but could not determine whether lin-28 acts upstream or downstream of lin-14 (Ambros 1989).

To examine the regulatory effect of lin-28 on lin-14, we tested the effect of a lin-28(n719) mutation on lin-14 protein accumulation. By genetic criteria, the lin28(n719) allele is a null mutant (V. Ambros, pers. comm.). By immunostaining, lin-28(n719) embryos show a pattern of $l i n-14$ protein accumulation very similar to wild type (Fig. 1d). In early L1 lin-28(n719) animals, lin-14 nuclear staining is normal in the ventral cord neurons, body wall muscle, and nerve ring cells, but hypodermal and intestinal nuclei show much weaker staining than wild type (Fig. 1d). By mid-Ll stage, lin-14 protein is very faint in all cell types of lin-28(n719) animals (data not shown). As in wild type, by the L2 stage lin-14 protein is undetectable in this mutant in all cell types and lineages (Fig. 1d).

These data suggest that lin-28 is not required for the initial expression of the lin-14 gene but is necessary for the continued expression or persistence during the Ll stage of 1 in-14 protein, particularly in hypodermal and intestinal cells.

lin-29 mutations do not affect the regulation of lin-14

lin-29 gene activity is necessary for a switch from L4 to adult hypodermal cell identities and, thus, only affects one of the temporal events regulated by lin-14 (Ambros and Horvitz 1984). However, the time at which the lin29-mediated switch occurs is regulated by lin-14: The L4-to-adult switch never occurs in lin-14(gf) mutants, and this switch occurs one stage precociously in lin14(1f) mutants (Ambros 1989). These genetic data predict that lin-29 is regulated by lin-14 so that mutations in lin-29 should have no effect on lin-14 regulation. Observation of the regulation of lin-14 expression in a lin-29 mutant confirmed this prediction. The lin-29(n333) mutant, which by genetic criteria appears to be null (V. Ambros, pers. comm.), displayed a lin-14 staining pattern similar to wild type in Ll stage animals and, as in wild type, displayed no lin-14 protein after L1 (Fig. 1e).

\section{lin-28 regulates lin-14 independently of lin-4 and the lin-14 negative regulatory element}

Because lin-4 and lin-14(gf) mutations result in higher levels of the lin-14 protein at post-L1 stages while lin-28 mutations have the opposite effect on regulation of lin14 protein level, we examined the temporal regulation of lin-14 in lin-28(n719); lin-4(e912) and lin-28(n719); lin14(n536gf) double mutants, both of which display a precocious phenotype as in lin-28 single mutants. Immunofluorescent analyses of embryos and early $\mathrm{L} 1$ stage larvae of both double mutants showed normal lin-14 protein staining (Fig. 2a,b). At L2 and later stages, the lin-14 protein is undetectable, as observed in lin-28(n719) but unlike lin-4 or lin-14(gf) mutants (Fig. 2a,b).

Thus, a functional lin-28 gene product is necessary for the high level of 1 in-14 protein normally observed at the L1 stage in wild-type animals and at all postembryonic stages in lin-14(gf) and lin-4 mutants. These data show that the lin-28-mediated positive regulation of lin-14 protein levels does not act via lin-4 or via the regulatory element in the lin-14 3' UTR that is deleted in the lin14(n536gf) mutant.
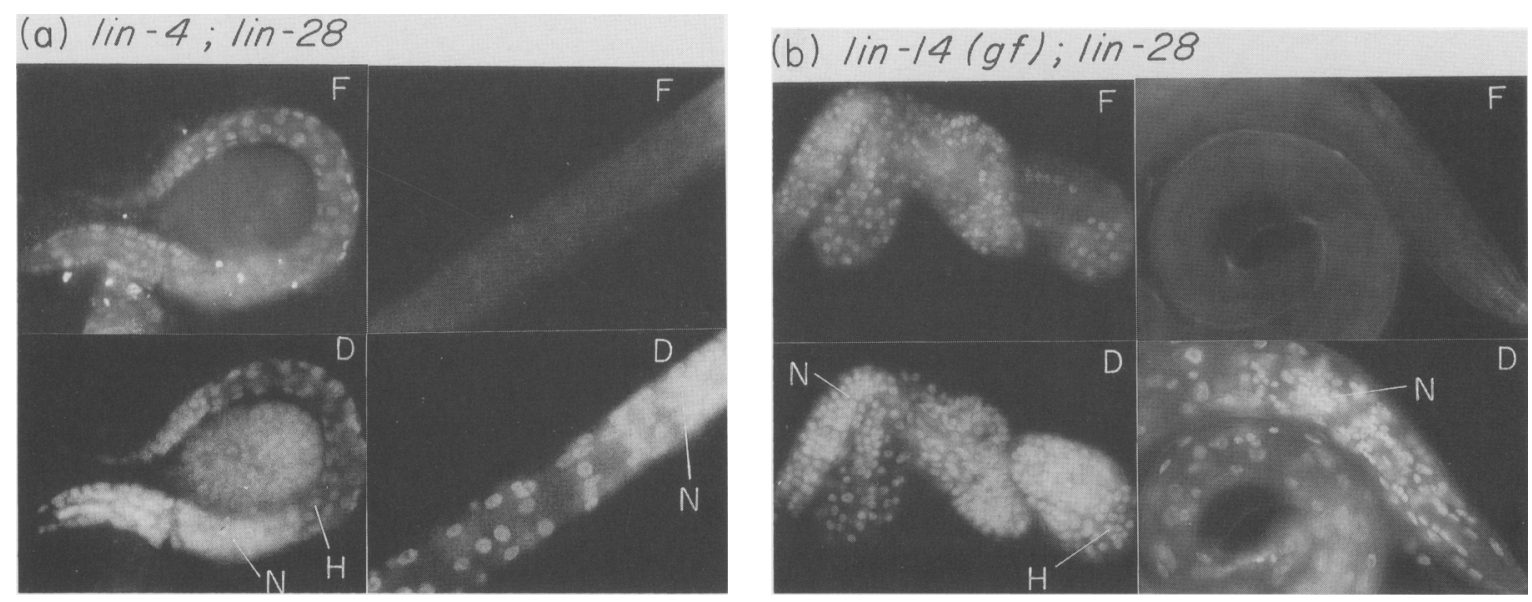

Figure 2. In situ immunofluorescent detection of lin-14 nuclear protein in double mutants. (a) (Top left) FITC; lin-4(e912); lin28(n719), showing an early Ll stage tenderly wrapped around an early embryo. lin-14-protein staining is not detectable in the early embryo, whereas the early Ll animal shows normal lin-14 protein staining. (Top right) FITC; lin-4; lin-28 L2 stage, showing no lin-14 protein at this stage, as with lin-28 mutants but unlike lin-4 mutant animals. (b) (Top left) FITC; lin-14(n536gf); lin-28(n719), showing newly hatched L1 and L2 late stage embryos, all of which accumulate lin-14 protein at these early stages. (Top right) FITC; L2 stage lin-14; lin-28 animal, showing no lin-14 protein at this stage. (See legend to Fig. 1 for abbreviations.) 
Down-regulation of lin-14 protein levels is triggered by developmental cues and not real time

lin-14 protein levels normally begin to decrease within a few hours of hatching during mid-Ll as postembryonic cell division is initiated. We investigated whether the down-regulation of 7 in-14 is dependent on the passage of time per se or on postembryonic developmental events by observing the regulation of $\operatorname{lin}-14$ protein in $\mathrm{L} 1$ animals in which development was suspended. When newly hatched L1 larvae are starved, they do not undergo postembryonic development but, instead, can remain suspended at this stage for up to 5 days (data not shown). Upon feeding, they resume development, executing normal L1-specific and subsequent cell lineages.

Immunofluorescent analysis of developmentally suspended L1 larvae at various time intervals of up to $125 \mathrm{hr}$ after hatching showed that there was no decline in the lin-14 protein levels as compared with early Ll stage animals (Fig. 3a). In comparison, during normal development, animals mature to adulthood in $\sim 60 \mathrm{hr}$ and have an undetectable level of 1 in-14 protein in nearly all cells within $15 \mathrm{hr}$ of hatching.

Ll larvae starved for 20,30, or $50 \mathrm{hr}$ were then fed for $12 \mathrm{hr}$. About $20 \%$ of the animals continued development into the L2 stage, and these animals showed a rapid decline in lin-14 protein levels similar to that occurring during normal development (Fig. 3b). Therefore, upon feeding, animals can resume postembryonic development and trigger the down-regulation of $\operatorname{lin}-14$ protein levels.

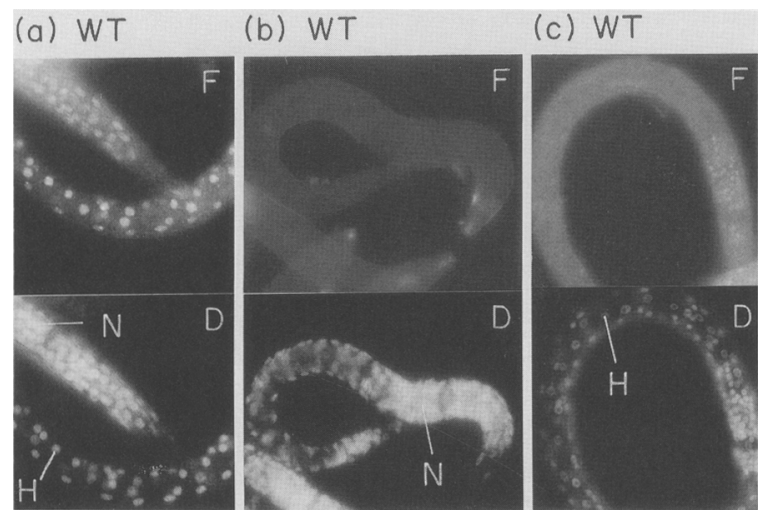

Figure 3. In situ immunofluorescent detection of lin-14 nuclear protein in starved wild-type animals. (Top) FITC; (bottom) DAPI. (a) Lateral view of wild-type Ll stage starved for $48 \mathrm{hr}$, showing lin-14 protein in hypodermal, nerve ring, and muscle nuclei. Animals starved up to $125 \mathrm{hr}$ were similarly arrested at the L1 stage and showed no variation in the pattern of lin-14 protein staining (data not shown). (b) Medial view of wild-type L1 stage starved in the presence of $1 \mathrm{mg} / \mathrm{ml}$ of cycloheximide for $48 \mathrm{hr}$, showing no detectable lin-14 protein. Animals treated in this manner were alive and still fairly mobile. $(c)$ Lateral view of wild-type late Ll stage, $12 \mathrm{hr}$ after feeding 48 -hr-starved L1 larvae. lin-14 protein is undetectable in hypodermal nuclei and barely detectable in nerve ring nuclei, similar to wild type (photo is overexposed to show weak lin-14 protein in neuronal nuclei). (See legend to Fig. 1 for abbreviations.)
We determined whether the presence of lin-14 protein in $\mathrm{Ll}$ animals at $48 \mathrm{hr}$ of starvation was due to stability of lin-14 protein or to continued translation of lin-14 mRNAs. After $24-48 \mathrm{hr}$ of starvation in $1 \mathrm{mg} / \mathrm{ml}$ of cycloheximide, animals were still alive; and after fixation, animals showed weak or no lin-14 protein staining compared with control animals (Fig. 3c). These animals showed no significant decrease in lin-14 transcript levels compared with control animals (data not shown). These data suggest that $1 \mathrm{in}-14$ protein or a protein that stabilizes lin-14 protein is continually translated in these developmentally arrested $\mathrm{L} 1$ animals.

\section{lin-28 is necessary for maintenance of lin-14 protein levels in developmentally arrested L1 larvae}

Because lin-14 protein levels remain elevated in developmentally arrested wild-type L1 animals, we examined whether a functional lin-28, lin-29, or lin-4 gene is necessary to maintain high levels of lin-14 protein in these animals. lin-28(n719) arrested L1 animals displayed little or no lin-14 protein staining after $48 \mathrm{hr}$ of starvation (Fig. 4a). These data suggest that lin-28 is necessary for the persistence or continued expression of lin-14 protein in suspended L1 larvae.

In contrast to lin-28 animals, lin-4(e912) mutant animals (Fig. 4b), lin-14(n355gf) mutants (data not shown), and lin-29 mutants (data not shown) displayed normal lin-14 protein levels and a normal cellular pattern of expression in developmentally arrested L1 larvae. Therefore, the lin-14 3'UTR, and lin-4 and lin-29 gene activities are not necessary to maintain high levels of lin-14 protein in arrested L1 animals. As in wild type, in the presence of cycloheximide, the lin-14 protein level in 48-hr-arrested lin-4 L1 larvae decreased to nearly undetectable (Fig. 4c). This suggests that the inappropriate accumulation of 1 in-14 protein in a lin-4 mutant is not caused by increased stability of 1 in- 14 protein in this mutant. Thus, the lin-4 gene activity is probably not functioning as (or regulating) a protease during the $\mathrm{Ll}$ stage.

lin-28; lin-4 and lin-28; lin-14(gf) double mutants, which normally express 1 in-14 protein only in the early L1 stage, showed no lin-14 protein staining after $48 \mathrm{hr}$ of starvation (data not shown), as with lin-28 mutant animals but unlike lin-4, lin-14(gf), or wild-type animals. These data show that the lin-28 gene activity is necessary for continued 7 in-14 protein accumulation in starved L1 animals even if the negative regulatory element in lin-14 is deleted or the lin-4 gene activity is missing.

\section{lin-28 and lin-14 do not control initiation of postembryonic development}

Normally, lin-14 protein levels fall just as postembryonic cell division begins during the L1 stage. Because

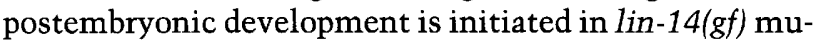
tants, although the level of 1 in-14 protein does not decrease normally, down-regulation of lin-14 protein is not necessary to initiate postembryonic development. To 
Figure 4. In situ immunofluorescent detection of lin-14 nuclear protein in starved heterochronic mutant animals. (Top) FITC; (bottom) DAPI. (a) Lateral view of lin-28(n719) Ll stage starved for $48 \mathrm{hr}$, showing no detectable lin-14 protein. In the presence of cycloheximide, lin-28 mutant animals similarly do not contain lin-14 protein (data not shown). (b) Medial view of lin-4(e912) Ll stage starved for $48 \mathrm{hr}$, showing lin-14 protein in nerve ring, muscle, intestinal, and ventral cord nuclei, as in wild-type $\mathrm{Ll}$ animals. $(c)$ Lateral view of lin-4(e912) Ll stage starved for $48 \mathrm{hr}$ in the presence of $1 \mathrm{mg} / \mathrm{ml}$ of cycloheximide, showing no detectable lin-14 protein. (See legend to Fig. 1 for abbreviations.)

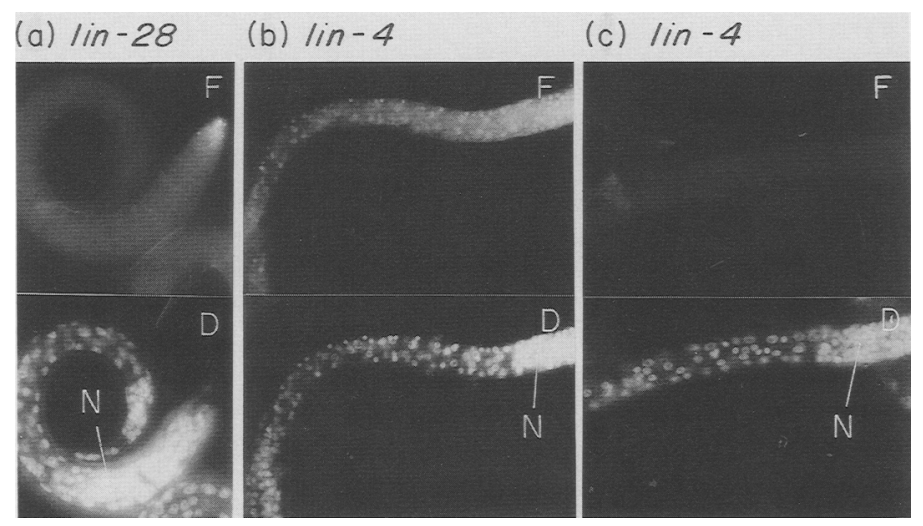

test whether decreased lin-14 protein levels are sufficient to initiate the postembryonic lineage, we observed whether postembryonic cell division occurs in starved lin-28 or lin-14(lf) mutant Ll larvae. We monitored whether postembryonic cell divisions occurred in the suspended lin-28(n719) (Fig. 4a) and lin-14(n179) (data not shown/ mutants. DAPI staining of fixed specimens was used to survey for production of the postembryonic Pn.a ventral cord neurons and Vn.a and Vn.p seam cells from the V1 to V6 lineages (Fig. 4a). The starved L1 larvae from these mutants displayed no postembryonic cell divisions after $48 \mathrm{hr}$, showing that development was suspended, although there was no lin-14 protein in these animals. These data show that lin-28 and lin-14 do not regulate the initiation of postembryonic cell division. Furthermore, the data show that lack of lin-28 gene activity does not down-regulate the lin-14 protein levels as a consequence of inappropriately initiating postembryonic cell division.

\section{Discussion}

The lin-14 protein gradient controls a major switch in the temporal pattern of the $C$. elegans cell lineage. The transition during the Ll stage from a high to a low level of lin-14 protein causes a number of blast cells to switch from L1-specific patterns of cell lineage during the early L1 stage to distinct L2-specific cell lineages that they execute at the next larval stage. Failure to down-regulate the lin-14 protein level after the L1 stage causes reiterations of L1-specific cell lineages (and lack of later cell lineages) at later stages, whereas failure to express high levels of lin-14 protein causes precocious expression of L2-specific cell lineages by blast cells at the L1 stage (Ambros and Horvitz 1987). We have shown here that the heterochronic genes $1 \mathrm{in}-4$ and $1 \mathrm{in}-28$ play a major role in the generation of the lin-14 temporal gradient, whereas lin-29 is not involved in establishing this gradient.

\section{lin-4 negatively regulates lin-14}

The lin-4(e912) mutation, which causes reiterations of L1-specific cell lineages at later larval stages, as with lin-14(gf) mutations, results in inappropriately high lev- els of lin-14 protein at L2, L3, and later developmental stages (Figs. 1 and 5). The lin-4 gene activity must therefore be necessary for the proper down-regulation of lin-14 protein levels.

Our results suggest that lin-4 may regulate lin-14 via the negative regulatory element in the 3' UTR of lin-14. Two lin-14(gf) mutants contain a rearrangement (n355) or a 607-bp deletion (n536) in the 3' UTR that abrogates the proper down-regulation of lin-14 protein at late larval stages (Wightman et al., this issue). The similarity of the lin-4 and lin-14(gf) phenotypes, both at the level of cell lineage changes and at the level of inappropriate lin-14 regulation, suggests that the lin-4 product may bind or activate a factor that binds to the lin-14 3' UTR to downregulate production of $l i n-14$ protein (Fig. 6). The simplest model for such a regulatory event is that the lin-4 gene product is expressed or activated during the $\mathrm{Ll}$ stage to bind directly or indirectly to the lin-14 mRNA which, in turn, down-regulates production of lin-14 protein during the late $\mathrm{L} 1$ stage (Fig. 6). The time at which lin- 4 acts has not been determined.

\section{lin-28 positively regulates lin-14}

Loss-of-function mutations in lin-28 are also heterochronic but affect only post-L1 stage hypodermal cell lineages. L1-specific lineages occur normally during the L1 stage in a lin-28(n719) null mutant; but at the L2 stage, hypodermal blast cells can express L3- or even L4-specific cell lineages (Fig. 5; Ambros and Horvitz 1984). In hypodermal blast cells, lin-28 gene activity is necessary for the reiterated L1 lineage at L2 and later stages caused by excess lin-14 gene activity in lin- 4 and lin-14(gf) mutants (Ambros 1989). This genetic result suggests that lin-28 may be necessary for the production of $l i n-14$ gene activity or may act downstream of $l i n-14$ in hypodermal blast cells. However, lin-28 affects fewer stage-specific events than lin-4 or lin-14. For example, the time of appearance of the alternative L3 dauer larval stage is controlled by lin-14 and lin-4 but not by lin-28 (Liu and Ambros 1989). These data suggested to Liu and Ambros that the heterochronic genes form a branched regulatory pathway. Such a branched pathway suggests that lin-4 and lin-14 could affect a broader number of cell types or act at a distinct time from lin-28. 

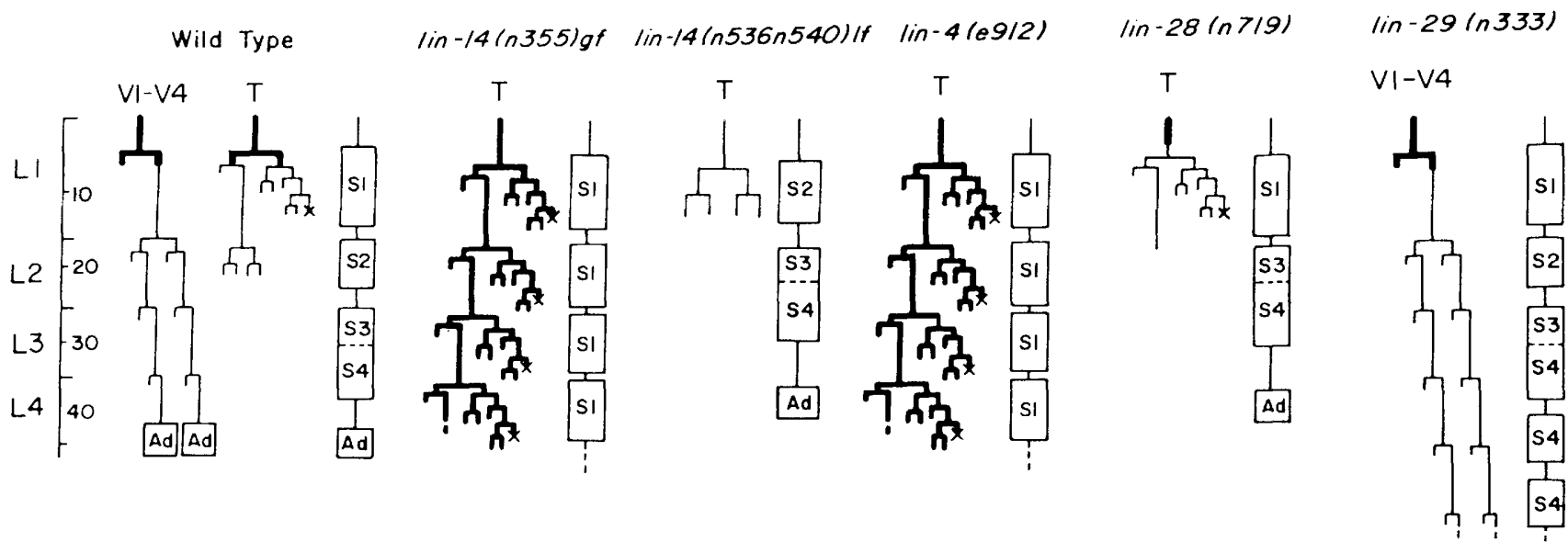

Figure 5. Summary of the temporal and cellular expression profile of lin-14 protein in wild-type, lin-14(gf), lin-14(lf), lin-4, lin-28, and lin-29 heterochronic mutants. Shown are the lineage changes induced by these mutations, and superimposed in dark line are those cells that also express the lin-14 protein. Wild type expresses lin-14 protein only during the Ll stage. lin-14(n355gf) and lin-4(e912) express the lin-14 protein at all developmental stages and, therefore, reiterate L1-specific cell lineages. lin-14(n536n540) shows no detectable lin-14 protein so that precocious L2-specific lineages occur during the L1 stage. lin-28(n 719) shows lin-14 protein only during the early L1 stage, albeit weakly, but not after it, so that a normal L1-specific lineage occurs, but L3 and later specific lineages occur precociously during the L2 stage. lin-29(n333) expresses lin-14 normally during the L1 stage so that L1-specific lineages and subsequent L2-, L3-, and L4-specific lineages are normally expressed. This mutant fails to express adult-specific traits and reiterates L4-specific cell identities regardless of whether lin-14 is mutant or not.

Analysis of the temporal regulation of lin-14 protein levels in a lin-28 null mutant showed that lin-28 either regulates lin-14 gene expression positively or is required for the stability of the lin-14 protein. The temporal and cellular profile of lin-14 protein accumulation begins normally in the lin-28(n719) mutant, but by the early $\mathrm{L} 1$ stage the level of $l i n-14$ protein is decreased relative to wild type in the hypodermal and intestinal cell lineages but not in neuronal and muscle cells (Fig. 1d). Although the difference in cell type expression of lin-14 observed in lin-28 mutants could account for the difference in lin-28 and lin-14 phenotypes with respect to dauer formation, other observations show that lin-28 affects lin14 protein levels in all cell types. For example, unlike wild type, the lin-14 protein level declines rapidly in all cell types of lin-28 L1 larvae that are developmentally arrested by starvation (Fig. 4). In addition, lin-28 gene activity is necessary for the inappropriate high level of lin-14 protein in all cells of post-Ll stage lin-4 and lin14(gf) mutants. These data rule out a simple model where lin-28 positively regulates lin-14 only in a specific subset of cells.

The temperature-sensitive period for the lin-14-mediated specification of L1-specific cell lineages in the hypodermis is just before those lineages occur during the early Ll stage (Ambros and Horvitz 1987). In lin-28 mutants, lin-14 protein levels begin to fall in hypodermal cells during the time that the gene must function to specify L1-specific cell lineages. Because lin-28 mutants execute normal L1-specific cell lineages, either the falling level of $l i n-14$ protein in these blast cells is sufficient to specify L1-specific cell lineages in those cells or lin-14 specifies L1-specific cell lineages to the hypodermal blast cells non-cell-autonomously.
Although these data suggest that lin-28 plays a role in the regulation of $l i n-14$, other observations argue that lin-28 also functions independently of lin-14. At the L2 stage, some hypodermal blast cells in lin-28 mutants execute L4-specific fates, whereas lin-14(lf) null mutants execute L3-specific fates (Ambros and Horvitz 1984). That is, at L2 and later stages, lin-28 mutants are more precocious than $l i n-14(l f)$ mutants. Furthermore, lin28; lin-14(lf) double mutants execute L4-specific fates during the L2 stage more often than either single mutant does (Ambros 1989). Therefore, lin-28 probably has functions in addition to regulation of the lin-14 protein.

\section{lin-4 and lin-28 antagonistically regulate lin-14}

lin- 4 and lin-28 act antagonistically to set the level of the lin-14 protein during the Ll stage and later (Fig. 6). Immunofluorescence analyses of lin-28; Iin-4 and lin28; 1 in-14(gf) double mutants showed that in both cases, accumulation of 1 in-14 protein at post-L1 stages depends on lin-28 activity (Fig. 2). These results are consistent with the genetic epistasis of lin-28 to lin-4 and lin-14(gf) mutations (Ambros 1989). These data distinguished between two models for down-regulation of lin-14 during the L1 stage in wild type: (1) During the L1 stage, lin-4 down-regulates 1 in-28 which, in turn, fails to up-regulate lin-14; or (2) lin-28 positively regulates lin-14 expression or stability during all postembryonic stages but lin-4mediated negative regulation normally overrides $\operatorname{lin}-28$ after the $\mathrm{L} 1$ stage. Because lin-28 is required for post-L1 stage accumulation of 1 in-14 protein even in lin-14(gf) mutants, which presumably have normal lin-4 gene activity, lin-28 must not be down-regulated by lin- 4 gene activity in this strain. We hypothesize that the logic is 


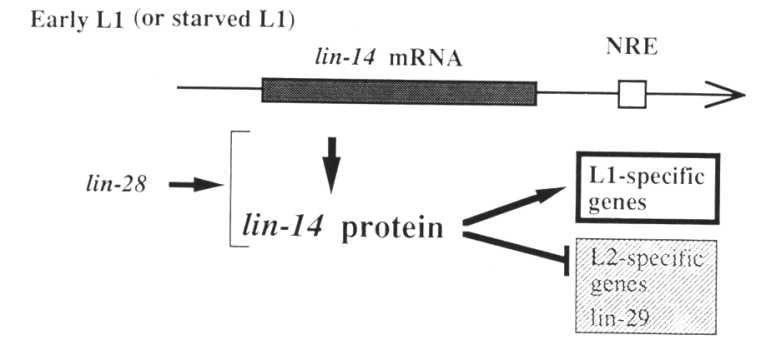

Late $\mathbf{L} 1$ after feeding

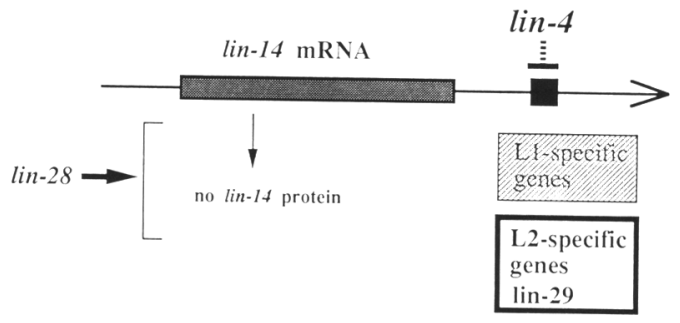

Figure 6. A model for the generation of the lin-14 protein gradient. In early Ll, or starved Ll, wild-type animals, lin-14 protein is actively translated and specifies L1-specific cell lineages, perhaps by activating $\mathrm{Ll}$-specific genes and/or repressing L2specific and later gene activities including lin-29. The normal temporal pattern of $l i n-14$ protein level is regulated by the heterochronic genes lin- 4 and $l i n-28$ that act antagonistically to define the lin-14 protein gradient. lin-28 is required either to activate lin-14 expression positively or to stabilize the lin-14 protein during the Ll stage of wild-type development and during L2 and later stages of development in lin-4 and lin-14(gf) mutants. A decrease in lin-14 protein levels during the mid-L1 stage is triggered by feeding and/or by postembryonic developmental events. This triggering event may activate lin- 4 expression or activity to regulate lin-14 negatively. The lin-4 product could bind to the lin-14 negative regulatory element (NRE) or regulate another factor that binds to the lin-14 NRE. The normal disappearance of the lin-14 protein at the L2 stage prevents the expression of L1-specific cell lineage genes and allows the expression of L2-specific and subsequent cell lineage genes, including lin-29, which specifies the switch from larval to adult stage cuticle formation (Ambros 1989). Arrows denote positive regulation; bars denote negative regulation.

the same in wild type: lin-4 does not act via lin-28 to down-regulate lin-14 during wild-type development either. The data also argue that $l i n-28$ positive regulation cannot be mediated by sequences in the lin-14 3' UTR deleted or disrupted by lin-14(gf) mutations because these mutants are still affected by lin-28. Rather, these data favor the second model that lin-28 positively regulates 1 in-14 protein levels independently from lin-4/lin14 3' UTR negative regulation.

\section{Down-regulation of lin-14 is triggered} by postembryonic developmental cues

We showed that the normal down-regulation of lin-14 protein level during the L 1 stage is not determined by the passage of a particular period of time from hatching. Rather lin-14 down-regulation is triggered by an unknown postembryonic event that can be blocked by starvation; wild-type $\mathrm{L} 1$ animals that are developmentally suspended at the L1 stage show no decline in lin-14 protein levels (Fig. 3). Treatment with cycloheximide showed that translation is necessary for maintenance of lin-14 protein and suggests that it is continually synthesized in these suspended L1 larvae (Fig. 3). lin-28 gene activity, but not lin-4 and lin-29, is necessary to maintain high levels of lin-14 protein before its down-regulation is triggered by feeding or postembryonic development. Upon feeding, animals that resume cell division and differentiation show the normal late L1 down-regulation of $\operatorname{lin}-14$ proteins (Fig. 3).

Down-regulation of Iin-14 protein levels does not cause initiation of postembryonic development, and the lin-28 mutation does not cause a decrease in the lin-14 protein level by initiating postembryonic development without the feeding cue. Starved Ll larvae from lin28(n719) and lin-14(n179) mutants did not initiate postembryonic cell lineages without feeding, although these animals had undetectable lin-14 protein levels (Fig. 3 ). Rather, these data suggest that feeding or the subsequent postembryonic development, or both, normally triggers the decrease in lin-14 protein levels. The feeding/postembryonic development switch could cause an increase in lin-4 activity to down-regulate the lin-14 protein level.

The postembryonic event that triggers lin-14 downregulation could be the same one that releases the animal for further cell divisions, or it is possible that downregulation of 1 in-14 depends on a signal from a particular cell generated from lineages early during the Ll stage. It is known that sensory detection of bacteria or the dauer pheromone antagonistically controls the dauer/nondauer switch during $C$. elegans development (Riddle 1988) and that specific neurons mediate this sensory/ developmental pathway (Bargmann and Horvitz 1991). Similarly, the same sensory neurons could control the initiation of postembryonic development and lin-14 down-regulation by feeding. On the other hand, simple increase in cell mass upon feeding could cause these changes. We are currently attempting to determine whether sensory detection of bacteria, ingestion of bacteria, or postembryonic development is necessary to trigger the lin-14 temporal switch and the postembryonic cell lineage.

\section{A model for the lin-14 temporal switch}

The cellular and temporal expression patterns of lin-14 proteins in wild-type and various heterochronic mutant strains have revealed aspects of how the lin-14 protein gradient is generated (Fig. 6). In wild-type late embryos and early Ll stage animals, lin-14 protein is actively translated. During the L1 stage, lin-28 either regulates lin-14 expression positively or stabilizes the lin-14 protein. The lin-14 protein specifies L1-specific cell lineages 
by activating L1-specific genes and/or repressing L2-specific genes and later genes such as lin-29.

The normal down-regulation of lin-14 protein is triggered by feeding and/or consequent postembryonic development early in the L1 stage. We suggest that lin- 4 or some other factor is activated during this time to downregulate lin-14 by the negative regulatory element in the 3' UTR. This factor could be activated by the sensation of food, or a specific cell generated or induced postembryonically may produce a signal that activates the factor.

Once the lin-4 and lin-28 genes are cloned, in situ expression studies will establish whether they are expressed in the same cells as 1 in-14 and at the same time as the lin-14 temporal switch. These studies will reveal whether these genes or their products are likely to interact directly with the lin-14 gene, mRNA, or protein. In vitro experiments will be necessary to show whether the protein products of these genes interact directly.

\section{Materials and methods}

\section{Strains}

C. elegans strains were propagated as defined by Brenner (1974). All heterochronic mutant strains were isolated as described by Ambros and Horvitz (1984, 1987).

\section{Immunofluorescence}

C. elegans wild type (N2) and mutant animals were fixed and immunostained with anti-lin-14 antibodies as described by Ruvkun and Giusto (1989), and animals were staged as described by Ambros and Horvitz (1987). Fluorescent nuclei were visualized with a Zeiss universal microscope as reported previously (Ruvkun and Guisto 1989). The lin-14 gene encodes multiple proteins with distinct amino-terminal regions (Wightman et al., this issue). The antibodies used in this study were raised against the lin-14A protein. Because the fusion used to raise antibodies includes a large portion of the domain common to all protein products and recognizes nothing in a lin-14 null mutant, it is likely that the antiserum recognizes all lin-14 protein products.

\section{Starvation}

Embryos were prepared by bleach treatment, washed thoroughly, and resuspended in S-basal medium (Wood et al. 1988). Developmentally arrested Ll larvae were fixed for immunostaining after $24,48,96$, or $125 \mathrm{hr}$ of incubation without a bacterial food source. Embryos were also incubated in medium containing $0.5,1$, or $5 \mathrm{mg} / \mathrm{ml}$ of cycloheximide (Sigma) under similar conditions of starvation. To monitor total protein synthesis, embryo preparations were hatched in the presence of $1 \mathrm{mCi} / \mathrm{ml}$ of $\left[{ }^{35} \mathrm{~S}\right]$ methionine medium containing $0,0.5$, or $5 \mathrm{mg} / \mathrm{ml}$ of cycloheximide; hatched L1 larvae were solubilized for protein gel analysis as described above. Total protein was displayed on Laemmli gels by Coomassie blue staining, and total counts incorporated were measured by excising the entire lane from the gel and counting ${ }^{35} \mathrm{~S}$. Translation was inhibited by $0 \%, 60 \%$, or $90 \%$, respectively, under these conditions.

\section{Acknowledgments}

We thank Victor Ambros and Shoshanna Gottlieb for numerous helpful discussions and members of the laboratory for sugges- tions on the manuscript. This work is supported by a grant from Hoechst A.G.

The publication costs of this article were defrayed in part by payment of page charges. This article must therefore be hereby marked "advertisement" in accordance with 18 USC section 1734 solely to indicate this fact.

\section{References}

Ambros, V. 1989. A hierarchy of regulatory genes controls a larva-to-adult developmental switch in C. elegans. Cell 57: 49-57.

Ambros, V. and H.R. Horvitz. 1984. Heterochronic mutants of the nematode Caenorhabditis elegans. Science 226: 409416.

. 1987. The lin-14 locus of Caenorhabditis elegans controls the time of expression of specific postembryonic developmental events. Genes \& Dev. 1: 398-414.

Bargmann, C.I. and H.R. Horvitz. 1991. Control of larval development by chemosensory neurons in Caenorhabditis elegans. Science 251: 1243-1246.

Brenner, S. 1974. The genetics of C. elegans. Genetics 77: 7194.

Chalfie, M., H.R. Horvitz, and J.E. Sulston. 1981. Mutations that lead to reiterations in the cell lineages of C. elegans. Cell 24: 59-69.

Horvitz, H.R. 1988. Genetics of cell lineage. In The nematode, Caenorhabditis elegans (ed. W.B. Wood), pp. 157-190. Cold Spring Harbor, Laboratory, Cold Spring Harbor, New York.

Ingham, P. 1988. The molecular genetics of embryonic pattern formation in Drosophila. Nature 335: 25-34.

Liu, Z. and V. Ambros. 1989. Heterochronic genes control the stage-specific initiation and expression of the dauer larva developmental program in Caenorhabditis elegans. Genes \& Dev. 3: 2039-2049.

Riddle, D.L. 1988. The dauer larva. In The nematode Caenorhabditis elegans (ed. W.B. Wood), pp. 393-412. Cold Spring Harbor Laboratory, Cold Spring Harbor, New York.

Ruvkun, G. and J. Giusto. 1989. The Caenorhabditis elegans heterochronic gene 1 in-14 encodes a nuclear protein that forms a temporal developmental switch. Nature 338: 313319.

Wood, W.B. (ed.) 1988. The nematode Caenorhabditis elegans. Cold Spring Harbor Laboratory, Cold Spring Harbor, New York. 


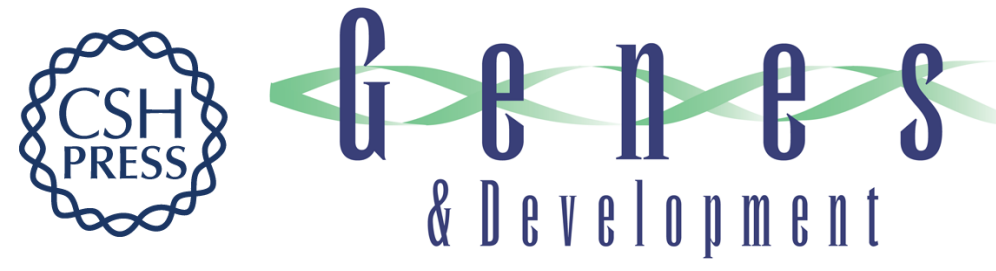

\section{Temporal regulation of lin-14 by the antagonistic action of two other heterochronic genes, lin-4 and lin-28.}

P Arasu, B Wightman and G Ruvkun

Genes Dev. 1991, 5:

Access the most recent version at doi:10.1101/gad.5.10.1825

References This article cites 9 articles, 5 of which can be accessed free at:

http://genesdev.cshlp.org/content/5/10/1825.full.html\#ref-list-1

License

Email Alerting

Service right corner of the article or click here.

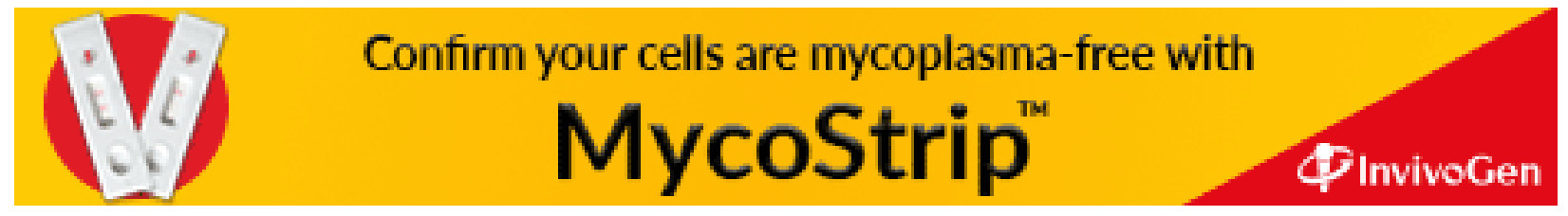

